\title{
The $\mathrm{N}$-acetylglucosaminyltransferase $\mathrm{V}$ inhibits the invasion of trophoblast cells by attenuating MMP2/9 activities in early human pregnancy
}

\author{
Abstract: \\ Objectives: Trophoblast invasion and migration is an essential part of \\ normal placentation and successful pregnancy. The process is \\ well-controlled by many factors at the fetal-maternal surface. Inadequate \\ trophoblast invasion may lead to poor placenta perfusion, or complications \\ such as preeclampsia (PE). There is accumulating evidence that \\ $\mathrm{N}$-acetylglucosaminyltransferase V (MGAT5) is correlated with tumor \\ invasion and metastasis. Our objective was to characterize MGAT5 \\ expression and function during placental development. \\ Methods: The expression of MGAT5 in human first trimester placental \\ tissue was determined by immunohistochemistry. To investigate whether \\ MGAT5 regulates trophoblast invasion and migration, we studied \\ invasion/migration of the HTR8/SVneo trophoblast cell line and used \\ human villous explants. Cell proliferation and apoptosis were measured by \\ CCK-8 assay and flow cytometric analysis respectively. The activities of \\ matrix metalloproteinase (MMP) 2/9, and the expression of tissue inhibitor \\ of metalloproteinase (TIMP) 1/2 were determined by gelatin zymography \\ and Western blotting respectively.


Results: MGAT5 was specifically localized in cytotrophblast, syncytiotrophoblast and trophoblast columns of human placental villi, decidual cells and some extravillous cells in the maternal decidua. MGAT5 shRNA significantly enhanced HTR8/SVneo cell invasion and migration, and increased villous explant outgrowth without affecting trophoblast proliferation and apoptosis. The enhanced effect of MGAT5 shRNA on trophoblast cell invasion was associated with increased gelatinolytic activities of MMP2/9 and decreased expression of TIMP1/2. Discussion and conclusion: Our data support a role of MGAT5 in inhibiting human trophoblast cell invasion and migration during early pregnancy by directly or indirectly regulation of MMP2/9 activities.

Keywords: MGAT5, placenta, trophoblast invasion, trophoblast migration, MMPs 


\section{Introduction}

Trophoblast cell invasion is a crucial progress for implantation, placentation and a successful pregnancy $[1,2]$. In the early stage of placental development, cytotrophblast (CTB) cells rapidly proliferate and differentiate into syncytiotrophoblast (STB) and extravillious trophoblast (EVT), and EVTs then begin to invade into the surrounding maternal decidua, the capillary network and then arterioles and endometrial spiral arteries. The blood vessel walls are remodeled by this invasion at the maternal-fetal interface to provide sufficient nutrients and oxygen for the fetus $[3,4]$. Impaired invasion of trophoblast cells into the maternal tissue or insufficient uterine spiral artery remodeling may lead to preeclampsia (PE) $[2,5]$, intrauterine growth restriction[6] and other obstetric complications $[7,8]$. The detailed molecular mechanism involved in regulating trophoblast invasion requires further elucidation.

There are several similarities between the behavior of placental cells and that of malignant tumor cells, such as invasion and growth characteristics [9]. However, the invasion of trophoblast is self-limited and is precisely regulated by the placenta-specific genes. This regulation ensures that invasion only occurs in early pregnancy and that it is restricted to the upper third of the myometrium $[10,11]$. A number of factors are involved in this process [12]; for example, CTBs secrete matrix metalloproteinases (MMPs) and its specific tissue inhibitor TIMPs. MMPs 
and TIMPs remain in a dynamic balance and tightly control the invasion of EVTs into maternal endomyometrium $[9,13,14]$.

Changes in glycan are associated with altered cell behaviors, and can influence including adhesion, migration, growth, and differentiation $[15,16]$. Glycosylation reactions are catalyzed by the glycosyltransferases, including $\mathrm{N}$-acetylglucosaminyltransferase V (MGAT5), also known as GnT-V, which plays a regulatory role in the synthesis of complex $\mathrm{N}$-glycans; this process is highly conserved in mammals $[17,18]$. It has been reported that an aberrant glycosylation induced by MGAT5 is associated with tumor malignant transformation, including tumor invasion and metastasis. Enhanced activity of MGAT5, resulting in an increase of B 1-6-branched oligosaccharides on the cell-surface, may contribute to the increased invasive potential of tumor cells [19]. Upregulated MGAT5 activity has been reported in human breast cancer, hepatocarcinoma and colon cancer [20-22]. Study of MGAT5 knockout mice showed decreased invasion and metastasis of the mammary tumors in vivo [23], suggesting that inhibitors of MGAT5 may have potential in the treatment of tumors. Furthermore, a higher expression of MGAT5 in first trimester placentas than those at term was determined [24].

Although highly expressed in placenta, the role of MGAT5 in human trophoblast function has not been characterised. Our objective was to investigate our hypothesis that MGAT5 is involved in trophoblast 
migration and invasion. We also studied the effects of MGAT5 on MMP2/9 activity and expression of TIMP1/2.

\section{Materials and Methods}

\subsection{Tissues collection}

First trimester human placental tissues $(5-10$ weeks gestation, $n=11)$ were obtained from healthy pregnant women undergoing elective termination of pregnancy for nonmedical reasons. Informed consent was obtained from all of the participating patients. Ethics approval was granted by the Ethics Committee of the First Affiliated Hospital of Chongqing Medical University.

Tissue samples were transported to the laboratory immediately after surgery. For Western blotting, tissues were washed three times with ice-cold phosphate-buffered saline (PBS), stored in liquid-nitrogen jar-freezing and kept at $-80^{\circ} \mathrm{C}$ until use. For immunohistochemistry, samples were washed with ice-cold PBS and fixed with 4\% neutral paraformaldehyde, followed with gradient ethanol dehydration and embedding in paraffin. For explant culture, samples were cut into $1-5 \mathrm{~mm}^{3}$, washed three times with ice-cold $0.9 \%$ saline and cultured immediately (see details in explant culture method).

\subsection{Immunohistochemistry (IHC)}


IHC was performed as previously described [26]. Sections of villi and decidua were deparaffinized and rehydrated in gradients of ethanol. Slides were boiled in citrate buffer $(10 \mathrm{mM}$ sodium citrate, $10 \mathrm{mM}$ citric acid, $\mathrm{pH}$ 6.0) in a microwave oven at $92-98^{\circ} \mathrm{C}$ for $10-15$ min to retrieve antigen. After blocking with $20 \%$ normal goat serum for $30 \mathrm{~min}$, sections were incubated with a primary MGAT5 antibody (1:400; NOUVS), cytokeratin7 (CK7) antibody(1:200; Abgent) or human leukocyte antigen G (HLA-G) antibody (1:400; Abcam) overnight at $4^{\circ} \mathrm{C}$. MGAT5 treated sections were then incubated with horseradishperoxidase(HRP) -conjugated goat anti-mouse IgG antibody (1:1000; Santa Cruz); the CK-7 and HLA-G sections were incubated with HRP conjugated goat anti-rabbit IgG antibody (1:1000; Santa Cruz) for $1 \mathrm{~h}$ at $37^{\circ} \mathrm{Cafter}$ washing. Sections were detected by diaminobenzidine substrate (Dakocytomation, California) working reagent for 20-50s and counterstained with hematoxylin.

\subsection{Cell culture and RNA Interference targeting MGAT5 in HTR8/SVneo} cells.

The HTR8/SVneo cell line, derived from human first trimester invasive EVT, is widely used in models of trophoblast invasion and migration. HTR8/SVneo cells used in this study were a kind gift from Dr. Charles Graham (Queen's University, Kingston, Ontario, Canada), as previously mentioned [27]. Cells were routinely cultured in RPMI-1640 
medium (Gibco) with $10 \%$ fetal bovine serum (FBS) at $37^{\circ} \mathrm{C}$ in an incubator with $5 \% \mathrm{CO}_{2}$. For gelatinolytic zymography, cells were cultured in serum-free media. According to the manufacturer's protocol, when the cells reached $70-80 \%$ confluence, the cells were transfected with a lentivirus vector-based short hairpin RNA (shRNA) directed against the sequence of MGAT5 (MGAT5 shRNA) [28] or with a negative control (scrambled shRNA) (GenePHarma, Shanghai, China). After screening using puromycin, a stable HTR8/ SVneo cell line with the silenced human MGAT5 gene was obtained and then used for the subsequent experiments.

\subsection{Immunofluorescence}

HTR8/SVneo cells were subjected to indirect immunofluorescence staining as described previously [26]. The primary antibody used was the anti-MGAT5 antibody (1:100; NOVOUS). The secondary antibody used was a fluorescein isothiocyanate-conjugated goat anti-mouse antibody (1:50; Santa Cruz Biotechnology). Nuclei were stained with propidium iodide ( $3 \mathrm{mg} / \mathrm{mL})$ dye. Images were acquired with an Olympus BMX-60 microscope equipped with a cooled charge-coupled device sensicamera (Cooke, Auburn Hills, Michigan) and Slidebook software (Intelligent Imaging Innovations, Denver, Colorado).

\subsection{Western blotting}


To detect expression of proteins, the placental villous explants and the HTR8/SVneo cells (human MGAT5 knockdown cells or negative control cells) were lysed in RIPA lysis buffer (Sigma, USA) followed by Western blotting analyses as previously described [27]. The primary antibodies against the following proteins were used: MGAT5 (1:500; NOUVS), $\beta$ $-\operatorname{actin}\left(1: 1000 ;\right.$ Santa Cruz), and TIMP1/2 (1:1000; Santa Cruz) at $4{ }^{\circ} \mathrm{C}$ overnight and followed by incubation with HRP conjugated secondary antibody (1:2000; Santa Cruz). Immunoreactive signals were detected by using enhanced chemiluminescence reagents and analyzed by a Chemi-doc image analyzer (Bio-Rad).

2.6. Matrigel cell invasion assay and transwell migration assay.

For the cell invasion assay, transwell inserts (Costar, Cambridge, MA, USA) were pre-coated with Matrigel (BD Biosciences, San Jose, CA;1:9 dilution), as described previously [27]. For the cell migration assay, the transwell inserts were not pre-coated with Matrigel. A total of $1.0 \times 10^{5}$ HTR8/SVneo cells (MGAT5 shRNA group or scrambled shRNA group) in serum-free medium were plated in the upper chamber, whereas $600 \mathrm{ul}$ medium with $10 \%$ FBS was added to the lower compartment. After incubation for $24 \mathrm{~h}$, the cells in the upper chamber of the inserts were scrubbed by cotton swab. Then the inserts were fixed in methanol and stained with crystal violet. To estimate the number of invaded or migrated 
cells, cells on the other side of the insert were counted with use of a light microscope (Olympus IX51, Japan) in five random fields at a magnification of X200. Data are showed as the fold change compare to the negative control values.

\subsection{Cell proliferation assay}

Cell viability was measured by the Cell Counting Kit-8 (CCK-8) assay (Dojindo Molecular Technologies, Rockville, MD, United States). A total of $1.0 \times 10^{4}$ cells in 100 ul of serum-free medium were seeded in each well of the 96-well plates (3 wells in each group), and CCK-8 (10ul) was added at different time points, and incubated at $37^{\circ} \mathrm{C}$ for another $1 \mathrm{~h}$. Serum-free Medium (100ul) without cells was used as a negative control. The CCK8 assay was performed at the daily intervals from the first day to the third day after seeding, and optical density (OD) value was measured with a microplate reader (Bio-Rad, United States) at $450 \mathrm{~nm}$. All assays were performed in triplicate and repeated three times.

\subsection{Cell Apoptosis Assay}

To quantify the rate of apoptosis in HTR8/SVneo cells pre-treated by shRNA, Annexin V-FITC and PI Apoptosis Detection Kits (Key-Gen Biotech, Nanjing, China) were used as previously described [27]. In brief, HTR8/SVneo cells (scrambled shRNA or MGAT5 shRNA) were grown on 
a six-well plate and incubated for $48 \mathrm{~h}$. Cells were then washed twice with ice-cold PBS, collected and stained in the Annexin V-FITC and PI binding buffer for 20min. The stained cells were analyzed by a FACS Vantage SE flow cytometer (BD Biosciences, San Jose, Calif., USA).

\subsection{Villous explant cultures}

Placental villous tissues $\left(1-5 \mathrm{~mm}^{3}\right)$ were dissected from the tips of villi and explanted as described previously $[27,29]$. Serum-free DMEM/F12 (Gibco) medium with 500nM shRNA targeting MGAT5 or an equal concentration of the scrambled shRNA was added into wells and incubated under low oxygen tension $(3 \% \mathrm{O} 2)$ for $72 \mathrm{~h}$ before RNA and protein collection. The outgrowth and migration of EVT from the distal end of the villous tips were recorded daily for up to 3 days. The EVT migration distance (from the cell column base to the outgrowth tip) was measured by Image $\mathbf{J}$ software. The explant experiment was quadruplicated and repeated five times.

\subsection{Gelatin zymography}

The gelatinolytic activity was identified by gelatin zymographic analysis, as previously described [30, 31]. A total of $10 \mathrm{ml}$ protein from the conditioned medium of HTR8/SVneo cells or placental villous explants underwent electrophoresis. Gels were then washed twice in $2.5 \%$ Triton 
$\mathrm{X}-100$ in $50 \mathrm{mM}$ Trise- $\mathrm{HCl}$ for $30 \mathrm{~min}$ and incubated in calcium assay buffer at $37^{\circ} \mathrm{C}$ overnight. Finally, gels were stained with Coomassie Brilliant Blue in 50\% methanol and 10\% acetic acid and destained in $10 \%$ acetic acid. The gels were scanned by densitometry with use of CS Analyzer 3.0.

\subsection{Statistical analysis}

Data are shown as the mean \pm SEM. All statistical analyses were performed using GraphPad Prism (GraphPad Software, San Diego, California). Differences between two groups were analyzed by independent t-test assuming a Gaussian distribution. A value of $p<0.05$ was defined as statistically significant.

\section{Results}

3.1. MGAT5 was expressed in human first trimester placentas and extravillous trophoblast

To indentify the expression of MGAT5 in early placental tissue, the localization and distribution of MGAT5 was investigated in detailed by immunohistochemistry (Fig.1). MGAT5 protein was specifically localized in villous CTB, syncytiotrophoblast (STB) and trophoblast columns (TC) (Fig. 1A and 1E). In the maternal decidua, MGAT5 was expressed in the decidual cells (DC) (Figure 1C and 1G) and some extravillous cells (EVT) 
cells which were defined by human leukocyte antigen G (HLA-G) staining (Fig.1 D and 1H,). Immunofluoresence staining confirmed that MGAT5 protein was presented and expressed in HTR8/SVneo cell line (Fig.2 A).

Fig.1. Expression of MGAT5 in human first trimester villi and deciduas. A. MGAT5 protein localized in villous cytotrophoblasts (CTB), syncytiotrophoblasts (STB) and trophoblast column (TC) in human placental villi. B. Immunostaining of cytokeratin 7(CK7), as a marker for CTB and TC. C. MGAT5 was expressed in the glandular epithelium (GE) and some extravillous trophoblasts (EVT) in maternal decidua. D. Immunostaining for human leukocyte antigen $\mathrm{G}$

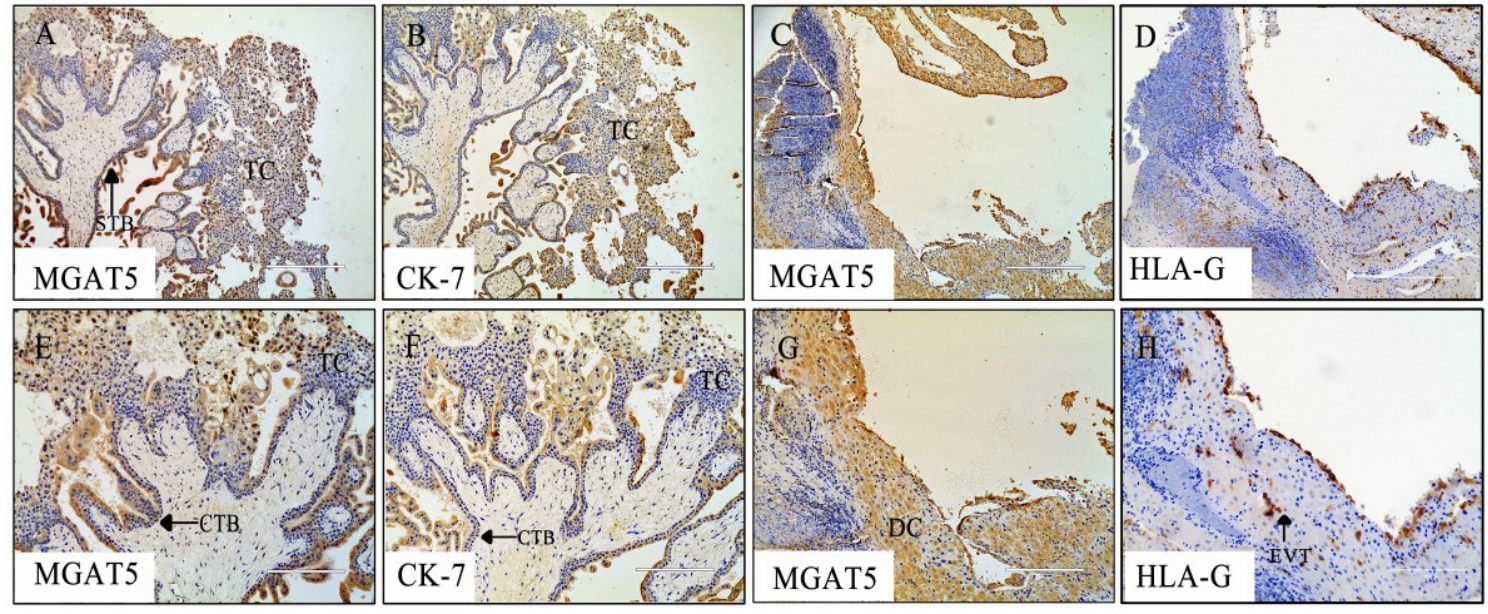

(HLA-G) as a marker of EVT in the maternal decidua. E-F. Higher powered images of A-B. G-H. Higher powered images of C-D. Fig. A-H are representative of $n=11$ villous and decidual samples. Scale bar, $100 \mu \mathrm{m}$.

3.2. MGAT5 shRNA significantly enhanced invasion and migration capacities of HTR8/SVneo cells

The expression of MGAT5 in trophoblast cell lines suggested a role in regulating cell behavior. To study whether MGAT5 knockdown had effects on cells migration and invasion, the cell model was subjected to transwell assay. 


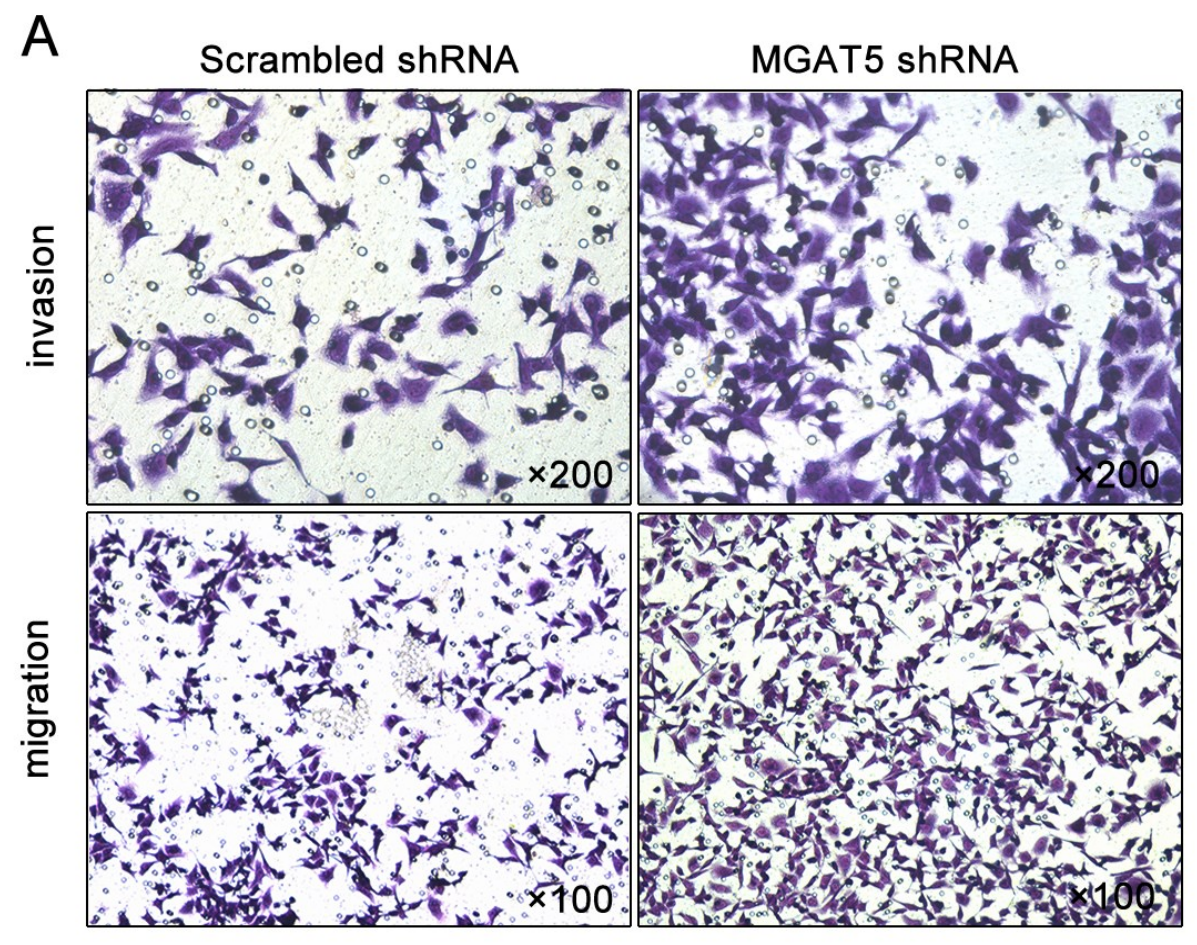

B

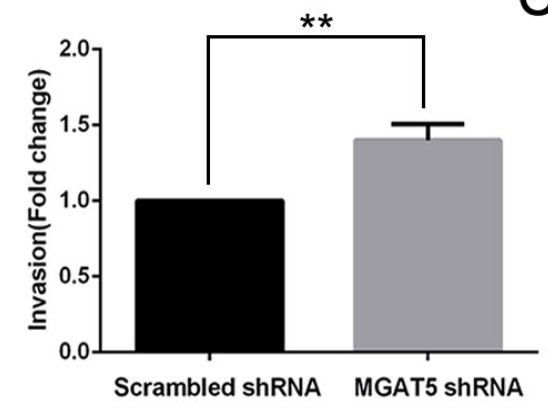

C

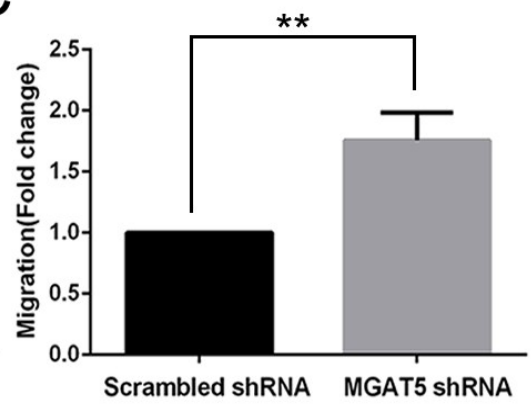

Fig.3. MGAT5 shRNA promoted invasion and migration of HTR8/SVneo cell. A.

HTR8/SVneo cells transfected with MGAT5 shRNA showed significantly increased migration and invasion potential compared to the scrambled shRNA control. Representative images of the Matrigel invasion assay $(\times 200)$ and transwell migration assay $(\times 100)$. B\&C. Statistical bar graphs showing the summary of three independent experiments of the Matrigel invasion assay and transwell migration assay. ( $t$-test; $* * P<0.01)$

In order to determine whether the increase of migrated and invaded cell number was a consequence of increased cell proliferation or/and 
decreased apoptosis, we further identified the effect of MGAT5 on cell proliferation and apoptosis. The MGAT5 knockdown had no significant effects on cell proliferation (Fig.4A) or apoptosis (Fig.4B) compared with the scrambled shRNA group. Taken together, in trophoblast, MGAT5 expression is negatively correlated with cell migration and invasion.
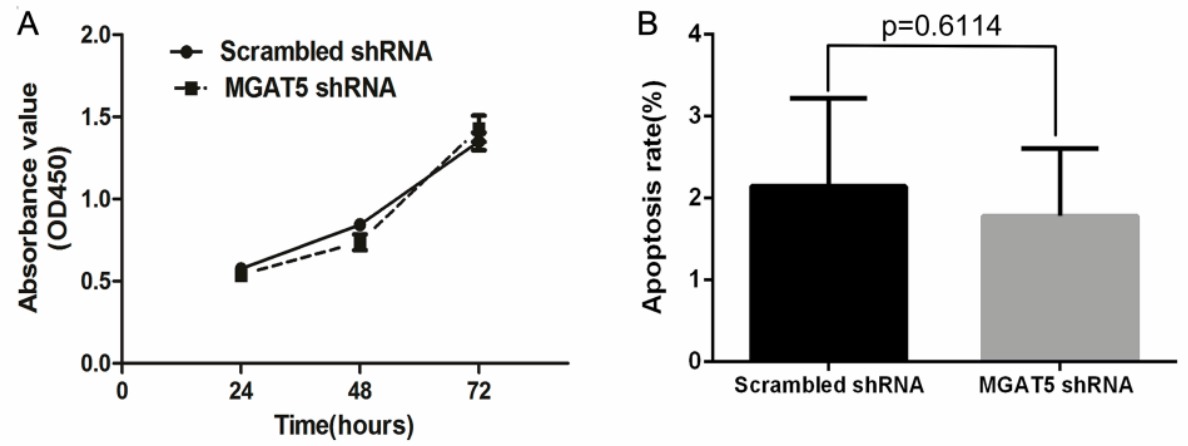

Fig.4. The effect of MGAT5 shRNA on apoptosis and proliferation of HTR8/SVneo cells. A. Proliferation assay of HTR8/SVneo cells ransfected with indicated shRNA over 3 days of culture. $(\mathrm{P}>0.05)$ B. Ratio of apoptotic cells in a HTR8/SVneo cell population transfected with indicated shRNA confirmed by FCM. ( $\mathrm{P}=0.6114)$

\subsection{Knockdown of MGAT5 promoted EVT migration in extravillous} explants

As MGAT5 was expressed in trophoblast of first trimester placentas, we next investigated the role of MGAT5 in trophoblast invasion and migration in vitro with the use of placental villous explants. The MGAT5 protein expression was significantly decreased by shRNA-meditated MGAT5 gene interference (Fig.5A and B). The outgrowth length as a result of EVT migration was measured at $24 \mathrm{~h}, 48 \mathrm{~h}$ and $72 \mathrm{~h}$. As shown in 
Figure.5C and D, the outgrowth distance in the MGAT5

shRNA-transfected group $(10054 \pm 565.9 \mu \mathrm{m})$ displayed a significant

increase compared with that of scrambled shRNA group $(5063 \pm 353.6 \mu \mathrm{m})$

$(* * P<0.01)$ after $72 \mathrm{~h}$ culture.

A

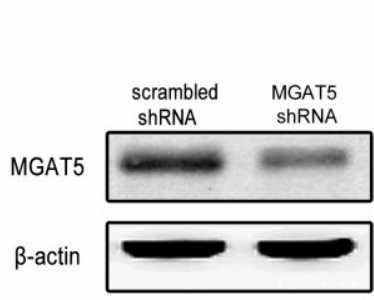

$\mathrm{D}$

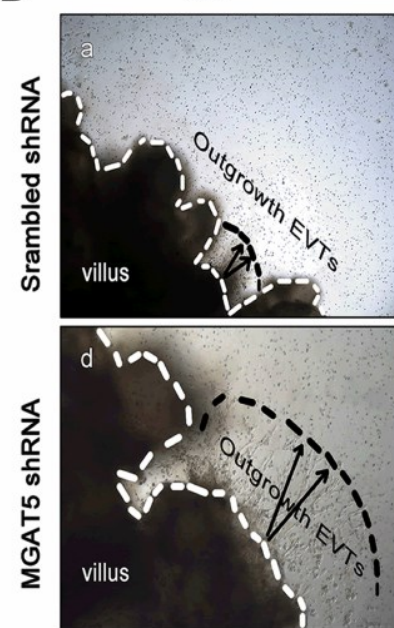

B

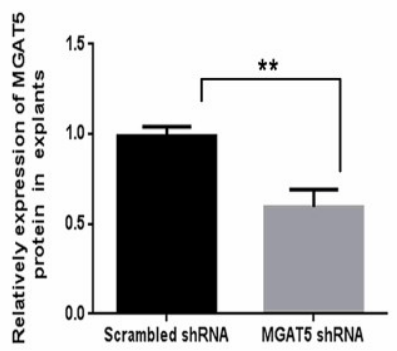

$48 \mathrm{~h}$

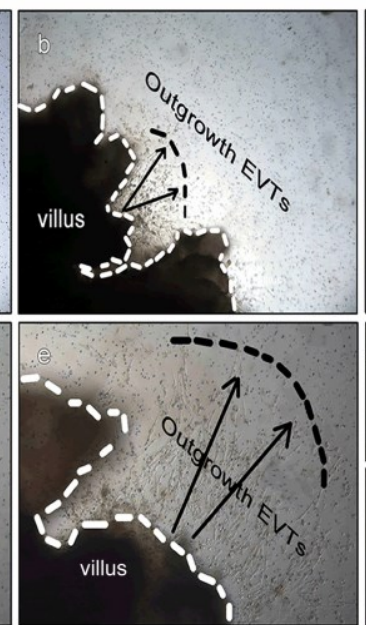

C

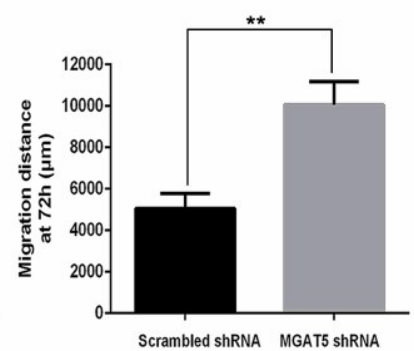

$72 \mathrm{~h}$

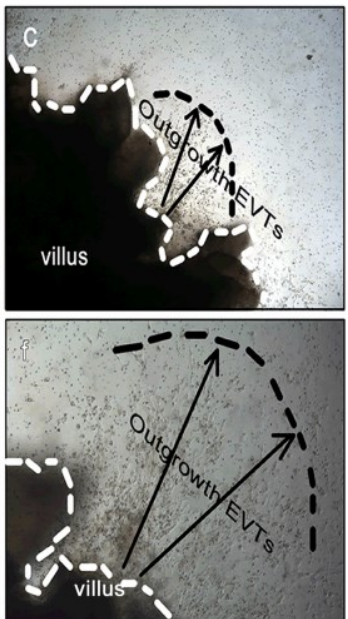

Fig.5. Knockdown of MGAT5 promoted the EVT outgrowth from the first trimester human placentas in an explant culture model. A. Confirming of RNA interference of MGAT5 was shown by western blotting. B. Statistical bar graphs showing ShRNA-mediated MGAT5 gene interference significantly reduced MGAT5 protein expression $(0.5940 \pm 0.04456$ compared with scrambled shRNA referenced at $1.00, * * P<0.01$ ) by western blot. C. Statistical analysis of the EVT outgrowth distance as representatively shown in D D. The outgrowth distance (indicated by the black arrows) of the explants under different treatments and different time durations. (Magnification $40 \times$ ).

3.4. The MGAT5 knockdown increased the activities of MMP1/2 and

This is the authors' final accepted manuscript, post peer review.

The version of record may be found at http://dx.doi.org/10.1016/j.placenta.2015.08.014 
attenuated the expression of TIMP $1 / 2$

According to previous reports, the MMP family plays a crucial role in digesting the extracellular matrix, and MMP2 and MMP9 are the major MMP forms expressed in placenta. TIMP1/2 are specific inhibitors of MMP2/9. To identify the gelatinolytic activities of MMP2 and MMP9 and expression of TIMP1 and TIMP2 in HTR8/SVneo cells and explants after MGAT5 shRNA transfection, gelatin zymography and Western blotting were performed. The zymography studies demonstrated that release of MMP2 and MMP9 was markedly increased in MGAT5 shRNA explants compare with the scrambled shRNA explants (Fig.6A and B, $* * P<0.01$ ), and expression of TIMP1 and TIMP2 was attenuated by MGAT5 knockdown in the villous explants (Fig.6A and $\mathrm{C}$; $* * P<0.01$ ). The MGAT5 knockdown in HTR8/SVneo cells (Fig. 6D) revealed that MGAT5 shRNA increased the activities of MMP9 and MMP2 $(* P<0.05)$ and decreased the expression of TIMP1 (Fig. 6E;*P<0.05) but not TIMP2 (Fig. 6E; $P>0.05$ ).

Fig.6. MGAT5 knockdown enhanced the gelatinolytic activities of MMP2/9 and decreased the expression of TIMP1/2. A. Representative image of gelatin zymography assay in placental villous explants. Western blots of TIMP1 and TIMP2 in placental villous explants. B. Statistical analysis of the gelatin zymographic results in A. C. Statistical analysis of the Western blotting results in A. Data was analyzed by $t$-test. D. Statistical analysis of the gelatin zymographic results in HTR8/SVneo cells. E. Statistical analysis of the Western blotting results in HTR8/SVneo cells.

\section{Discussion}

In the current study, we found that MGAT5 was expressed in the 
maternal deciduas and also localized in trophoblast cells; MGAT\% was implicated as having a crucial role in inhibiting trophoblast migration and invasion. Knockdown of MGAT5 resulted in enhanced cell migration and invasion in a trophoblast cell line HTR8/SVneo; this may reflect altered activities of MMP2/9 and expression of TIMP1/2.

Numerous studies have been confirmed a upregulation of MAGT5 in human tumors, such as breast cancer [20], colon carcinoma [22] and that upregulation of MGAT5 suggests favorable prognosis for bladder carcinoma [32], non-small cell lung cancer[33] and neuroblastoma[34]. But there have been few studies of MGAT5 expression and function in human early/term placental tissues. Aspects of trophoblast cell behavior parallel those of malignant tumor cells; we thus assumed that MGAT5 might also play an important role in placentation and cell invasion. Our data demonstrated that MGAT5 was expressed in cytotrophoblast, syncytiotrophoblast cells and trophoblast columns and in the decidua (decidual cells and some EVT cells). This finding suggested that MGAT5 is involved in trophoblast invasion and placentation. We further showed that MGAT5 knockdown enhanced the invasion and migration potential in the trophoblast cell line HTR8/SVneo without affecting cell apoptosis and proliferation, and that MGAT5 knockdown promoted the outgrowth and migration capacity of first trimester human placental villi in an in vitro explant culture. An advantage of the villous explant model, as compared to 
primary trophoblast cells, is that it both reflects the complicated relationship between different types of cells and maintains the integrity of the tissue structure [36]. Finally, upregulation of MMP2/9 and downregulation of TIMP1/2 were confirmed in the MGAT5 shRNA explants/cells.

Our data are inconsistent with previous studies in which knockdown of MGAT5 inhibited tumor cell invasion and migration [20-22], but are in accord with studies which used the Jar human choriocarcinoma cell line. [37] Such a conflict may result from the different type of cells or cell lines used in the experiments. Studies have investigated the role of MGAT5 in migration and invasion with the use of cancer cell lines; however, trophoblast is not a malignant cell type. The invasion of trophoblast is self-limited and regulated by placenta-specific genes,. This regulation ensures that the invasion of the EVT only occurs during early pregnancy and that it is restricted to the proximal third of the myometrium $[10,35]$.

The MMP family is considered to be key to tumor invasion, and high MMP9 expression is thought to be crucial for migration/invasion of endothelial cells into the adjacent stroma[13,14]. MMPs, especially MMP2 and 9 play critical roles in trophoblast invasion by remodeling the ECM. The molecular mechanisms underlying the N-glycan regulation of the invasive function of MMP9 in placenta need to be elucidated. Here, we found that MGAT5 specific RNA interference in the explants and 
HTR8/SVneo cells resulted in increased activities of MMP2/9, decreased expression of TIMP1/2, and the promotion of trophoblast migration and invasion. Our data indicate that the trophoblast cells share similar cell invasion mechanisms with tumor cells. In fact, it has been previously reported that TIMP1, as a target protein for MGAT5 in human colon cancer cell WiDr, and the aberrantly glycosylated TIMP1 showed the weaker inhibition on both MMP2/9, which was closely associated with cancer cell invasion and metastasis in vivo as well as in vitro [41, 42].

With regard to the downstream signaling pathway, it has been previously reported that aberrant branched sugar chains inhibit the clustering of integrin a $5 \beta 1$, composition of focal adhesion and phosphorylation of FAK; further studies are needed to confirm the exact signaling pathways whereby regulation of MGAT5 expression and/or function induces trophoblast migration and invasion..

MGAT5 is, an important glycosyltransferase involved in the formation of branching asparagine-linked oligosaccharides [38]. Integrins contain nearly $20-30$ consensus $\mathrm{N}$-glycosylation sites in both $\alpha-$ and $\beta$ -subunits, modifications of these $\mathrm{N}$-glycans on integrins have the potential to alter cell function and behavior [39]. It has been reported that a $5 \beta 1$ integrin is a MGAT5 target molecule and increased $\beta$ 1,6-N-acetylglucosamine ( $\beta$ 1-6GlcNAc) branching on $\beta 1$ integrin by 
GnT-V in inducing migration of neck squamous cell carcinoma [40]. So,

there may be another way of MGAT5 to regulate cell invasion and

migration, our further study would focus on the underlying relationship

between MGAT5 and a 5 B 1 integrin.

Whether MGAT5 knockdown can promote trophoblast function and

decrease the secretion of anti-angiogenesis factors in placental

insufficiency condition, such as preeclampsia or intrauterine growth

restriction remains to be determined. If so, MGAT5 may serve as a new

potential target for treating these pregnancy complications. We have

already found MGAT5 expression is elevated in placentas from

pregnancies complicated by preeclampsia [REFERENCE]; as compared to

placentas from normal pregnancies; further study should clarify the

relationship between MGAT5 and preeclampsia.

In summary, our present data demonstrated that MAGT5 is expressed

in trophoblast cells in human first trimester placenta, and that MAGT5

restricted the migration and invasion of trophoblast cells by inhibiting the activities of MMP2/9. Therefore, MGAT5 may be an important regulator during placentation.

\section{Conflict of interest}

The authors declare no conflict of interest. 


\section{zAcknowledgements}

This study was supported by the National Natural Science Foundation of China (No.81300508, 81300509 and 81471472) and the Research Fund for the Doctoral Program of Higher Education (No.2013550311003). 


\section{References}

[1] Red-Horse K, Zhou Y, Genbacev O, Prakobphol A, Foulk R, Mcmaster M, et al. Trophoblast differentiation during embryo implantation and formation of the maternal-fetal interface. J Clin Invest 2004;114(6):744-754.

[2] Zhou Y, Damsky C H, Chiu K, Roberts J M, Fisher S J. Preeclampsia is associated with abnormal expression of adhesion molecules by invasive cytotrophoblasts. J Clin Invest 1993;91(3):950-960.

[3] Bischof P, Meisser A, Campana A. Biochemistry and molecular biology of trophoblast invasion. Ann N Y Acad Sci 2001;943:157-162.

[4] Knofler M. Critical growth factors and signalling pathways controlling human trophoblast invasion. Int J Dev Biol 2010;54(2-3):269-280.

[5] Steegers E A, von Dadelszen P, Duvekot J J, Pijnenborg R. Pre-eclampsia. Lancet 2010;376(9741):631-644.

[6] Gerretsen G, Huisjes H J, Elema J D. Morphological changes of the spiral arteries in the placental bed in relation to pre-eclampsia and fetal growth retardation. Br J Obstet Gynaecol 1981;88(9):876-881.

[7] Hustin J, Jauniaux E, Schaaps J P. Histological study of the materno-embryonic interface in spontaneous abortion. Placenta 1990;11(6):477-486.

[8] Mcmaster-Fay R A. Failure of physiologic transformation of the spiral arteries of the uteroplacental circulation in patients with preterm labor and intact membranes. Am J Obstet Gynecol 2004;191(5):1837-1838, 1838-1839.

[9] Halasz M, Polgar B, Berta G, Czimbalek L, Szekeres-Bartho J. Progesterone-induced blocking factor differentially regulates trophoblast and tumor invasion by altering matrix metalloproteinase activity. Cell Mol Life Sci 2013;70(23):4617-4630.

[10] Strickland S, Richards W G. Invasion of the trophoblasts. Cell 1992;71(3):355-357.

[11] Staun-Ram E, Shalev E. Human trophoblast function during the implantation process. Reprod Biol Endocrinol 2005;3:56.

[12] Cohen M, Bischof P. Factors regulating trophoblast invasion. Gynecol Obstet Invest 2007;64(3):126-130.

This is the authors' final accepted manuscript, post peer review. The version of record may be found at http://dx.doi.org/10.1016/i.placenta.2015.08.014 
[13] Walter I, Boos A. Matrix metalloproteinases (MMP-2 and MMP-9) and tissue inhibitor-2 of matrix metalloproteinases (TIMP-2) in the placenta and interplacental uterine wall in normal cows and in cattle with retention of fetal membranes. Placenta 2001;22(5):473-483.

[14] Cohen M, Meisser A, Bischof P. Metalloproteinases and human placental invasiveness. Placenta 2006;27(8):783-793.

[15] Hakomori S. Aberrant glycosylation in tumors and tumor-associated carbohydrate antigens. Adv Cancer Res 1989;52:257-331.

[16] Hakomori S. Glycosylation defining cancer malignancy: new wine in an old bottle. Proc Natl Acad Sci U S a 2002;99(16):10231-10233.

[17] Lowe J B, Marth J D. A genetic approach to Mammalian glycan function. Annu Rev Biochem 2003;72:643-691.

[18] Chakraborty A K, Pawelek J M. GnT-V, macrophage and cancer metastasis: a common link. Clin Exp Metastasis 2003;20(4):365-373.

[19] Dennis J W, Laferte S, Waghorne C, Breitman M L, Kerbel R S. Beta 1-6 branching of Asn-linked oligosaccharides is directly associated with metastasis. Science 1987;236(4801):582-585.

[20] Li D, Li Y, Wu X, Li Q, Yu J, Gen J, et al. Knockdown of Mgat5 inhibits breast cancer cell growth with activation of CD4+ T cells and macrophages. J Immunol 2008;180(5):3158-3165.

[21] Guo P, Chen H J, Wang Q Y, Chen H L. Down regulation of N-acetylglucosaminyltransferase V facilitates all-transretinoic acid to induce apoptosis of human hepatocarcinoma cells. Mol Cell Biochem 2006;284(1-2):103-110

[22] Murata K, Miyoshi E, Kameyama M, Ishikawa O, Kabuto T, Sasaki Y, et al. Expression of $\mathrm{N}$-acetylglucosaminyltransferase $\mathrm{V}$ in colorectal cancer correlates with metastasis and poor prognosis. Clin Cancer Res 2000;6(5):1772-1777.

[23] Granovsky M, Fata J, Pawling J, Muller W J, Khokha R, Dennis J W. Suppression of tumor growth and metastasis in Mgat5-deficient mice. Nat Med 2000;6(3):306-312.

[24] Tomiie M, Isaka S, Miyoshi E, Taniguchi N, Kimura T, Ogita K, et al. Elevated expression of $\mathrm{N}$-acetylglucosaminyltransferase $\mathrm{V}$ in first trimester human placenta. Biochem Biophys Res Commun 2005;330(3):999-1004.

This is the authors' final accepted manuscript, post peer review. The version of record may be found at http://dx.doi.org/10.1016/j.placenta.2015.08.014 
[25] Yang Z, Bai B, Luo X, Xiao X, Liu X, Ding Y, et al. Downregulated Kruppel-like factor 8 is involved in decreased trophoblast invasion under hypoxia-reoxygenation conditions. Reprod Sci 2014;21(1):72-81

[26] Liu X, Mu H, Luo X, Xiao X, Ding Y, Yin N, et al. Expression of Gadd45alpha in human early placenta and its role in trophoblast invasion. Placenta 2014;35(6):370-377.

[27] Yang H M, Yu C, Yang Z. N-acetylglucosaminyltransferase V negatively regulates integrin alpha5beta1-mediated monocyte adhesion and transmigration through vascular endothelium. Int $\mathrm{J}$ Oncol 2012;41(2):589-598.

[28] Li W, Liu D, Chang W, Lu X, Wang Y L, Wang H, et al. Role of IGF2BP3 in trophoblast cell invasion and migration. Cell Death Dis 2014;5:e1025.

[29] Staun-Ram E, Goldman S, Gabarin D, Shalev E. Expression and importance of matrix metalloproteinase 2 and 9 (MMP-2 and -9) in human trophoblast invasion. Reprod Biol Endocrinol 2004;2:59.

[30] Wang Q, Rajshankar D, Laschinger C, Talior-Volodarsky I, Wang Y, Downey G P, et al. Importance of protein-tyrosine phosphatase-alpha catalytic domains for interactions with SHP-2 and interleukin-1-induced matrix metalloproteinase-3 expression. $\mathrm{J}$ Biol Chem 2010;285(29):22308-22317.

[31] Ishimura $\mathrm{H}$, Takahashi $\mathrm{T}$, Nakagawa $\mathrm{H}$, Nishimura $\mathrm{S}$, Arai $\mathrm{Y}$, Horikawa $\mathrm{Y}$, et al. $\mathrm{N}$-acetylglucosaminyltransferase $\mathrm{V}$ and beta 1-6 branching $\mathrm{N}$-linked oligosaccharides are associated with good prognosis of patients with bladder cancer. Clin Cancer Res 2006;12(8):2506-2511.

[32] Dosaka-Akita H, Miyoshi E, Suzuki O, Itoh T, Katoh H, Taniguchi N. Expression of $\mathrm{N}$-acetylglucosaminyltransferase $\mathrm{v}$ is associated with prognosis and histology in non-small cell lung cancers. Clin Cancer Res 2004;10(5):1773-1779.

[33] Inamori K, Gu J, Ohira M, Kawasaki A, Nakamura Y, Nakagawa T, et al. High expression of $\mathrm{N}$-acetylglucosaminyltransferase $\mathrm{V}$ in favorable neuroblastomas: Involvement of its effect on apoptosis. FEBS Lett 2006;580(2):627-632.

[34] Newby D, Marks L, Cousins F, Duffie E, Lyall F. Villous explant culture: characterization and evaluation of a model to study trophoblast invasion. Hypertens Pregnancy 2005;24(1):75-91.

[35] Yamamoto E, Ino K, Miyoshi E, Inamori K, Abe A, Sumigama S, et al. 
$\mathrm{N}$-acetylglucosaminyltransferase $\mathrm{V}$ regulates extravillous trophoblast invasion through glycosylation of alpha5beta1 integrin. Endocrinology 2009;150(2):990-999.

[36] Lee J H, Kang J G, Song K J, Jeon S K, Oh S, Kim Y S, et al. N-Acetylglucosaminyltransferase $\mathrm{V}$ triggers overexpression of MT1-MMP and reinforces the invasive/metastatic potential of cancer cells. Biochem Biophys Res Commun 2013;431(4):658-663.

[37] Kim Y S, Hwang S Y, Kang H Y, Sohn H, Oh S, Kim J Y, et al. Functional proteomics study reveals that $\mathrm{N}$-Acetylglucosaminyltransferase $\mathrm{V}$ reinforces the invasive/metastatic potential of colon cancer through aberrant glycosylation on tissue inhibitor of metalloproteinase-1. Mol Cell Proteomics 2008;7(1):1-14.

[38] Brockhausen I, Narasimhan S, Schachter H. The biosynthesis of highly branched N-glycans: studies on the sequential pathway and functional role of $\mathrm{N}$-acetylglucosaminyltransferases I, II, III, IV, V and VI. Biochimie 1988;70(11):1521-1533.

[39] Gu J, Taniguchi N. Regulation of integrin functions by N-glycans. Glycoconj J 2004;21(1-2):9-15.

[40] Guo H B, Lee I, Kamar M, Akiyama S K, Pierce M. Aberrant N-glycosylation of beta1 integrin causes reduced alpha5beta1 integrin clustering and stimulates cell migration. Cancer Res 2002;62(23):6837-6845 